\title{
A sheet flow model to estimate the sediment transport rate for beaches backed by seawalls
}

\author{
M. Shakeri Majd ${ }^{1}$, M. A. Lashteh Neshaei ${ }^{2} \&$ M. Taleghani ${ }^{3}$ \\ ${ }^{1}$ Department of Civil Engineering, Islamic Azad University - Anzali Unit, \\ Iran \\ ${ }^{2}$ Department of Civil Engineering, Guilan University, Iran \\ ${ }^{3}$ Department of Management, Islamic Azad University - Rasht Unit, Iran
}

\begin{abstract}
To consider the effect of reflective structures on coastal sediment transport and beach morphology, experiments have been performed at laboratory model scale on a partially reflective seawall located in the surf zone. The main objectives of these experiments were to measure beach profile evolution on an open beach with two different sizes of sand and to compare the results with those on a beach fronting a partially reflective seawall. The results obtained from the profile evolution measurements in front of reflective seawalls indicate that the extent to which a seawall affects the processes on the fronting beaches largely depends on its location relative to the active shore face. A seawall located well landward of the active shore face will behave in much the same way as a natural beach whereas seawalls located in the active shore face will modify the near-shore beach profile because of the effects of reflected waves. A simple sheet flow model, based on the measured probability density functions of near-bed horizontal velocities, is developed to predict the short-term response of a partially reflective structure to random wave attack. The main conceptual innovation of the model is taking the probability density functions of the velocities into account and integrating them to calculate the sediment displacements across the profile using a threshold criterion for initiation of sediment motion. The results obtained from the model and comparison with the experimental results are promising and encouraging for further developments of the preliminary model.

Keywords: coastal sediment transport rate, beach morphology, Probabilistic Model, seawalls effects.
\end{abstract}




\section{Introduction}

A number of researchers have shown that the beach change near seawalls, both in magnitude and temporal variation is similar to that on beaches without seawalls, if a sediment supply exists. The physical processes of the seawall and beach interaction, however, must be well understood in order to assess the relative performance of seawalls and alternative shore protection methods. Clearly, investigations of these processes demands measured data for an arbitrary beach profile, which can be acquired by performing laboratory-scale experiments in both two and three dimensions. Clearly, there is insufficient theoretical work on hydrodynamics of the surf zone and the associated sediment transportation in the vicinity of reflective structures (Rakha and Kamphuis [1]). In the present study, based on the results obtained from the experiments, a semi-empirical model has been developed to estimate the short-term response of a beach to a partially reflective structure located in the surf zone. According to the available literature and with regard to the long-term processes, beaches are thought to behave similarly irrespective of the presence of a reflective structure (Kamphuis et al [2]). However, the present investigations show a remarkably different behavior of the beach in the presence of a partially reflective structure in shortterm storm events. It is to be emphasized that the present work is focused on the cross-shore coastal hydrodynamics, sediment transport and profile evolution. Hence, it is not surprising to observe a different behavior of the beach in front of the structure. In particular, the application of a sheet flow model to calculate the sediment transport in the surf zone is considered in the present study. Therefore, attention is focused on the sheet flow regime of sediment movement close to the bed, neglecting the sediment suspension. The results obtained from the experimental investigations and model development in this respect are classified and discussed in detail.

\section{Experiments}

\subsection{Experimental apparatus and procedure}

To measure the sediment transport rate inside the surf zone, laboratory experiments were performed in a large two-dimensional wave tank at Imperial College Hydraulics Laboratory. Fig. 1 illustrates the experimental set up. Using a typical JONSWAP spectrum, irregular waves were generated at one end of the tank and the bed level changes were measured using two different sizes of sediments (fine sand, $\mathrm{D}_{50}=0.5 \mathrm{~mm}$ and coarse sand, $\mathrm{D}_{50}=1.5 \mathrm{~mm}$ ) on a natural beach (Holmes et al [3]).

The experiments were then repeated in front of the partially reflective structure located in the surf zone. The sediment sizes were chosen so that the threshold of motion was exceeded for a significant period of time. The bed elevations were measured after each 30, 60,120 and 240 minutes of wave action. It was assumed that after 4 hours of wave action, the profile will approach an equilibrium condition. It has to be noted that the experiments were focused on 
the short-term beach morphology, i.e. storm events. Hence, selecting 240 minutes wave action assuming a 1:30 scale, simulating $240 \times \sqrt{\mathbf{3 0}}=22$ hours at full-scale, was felt to be reasonable to create a stable profile.

\subsection{Measurement of beach profile evolution on an open beach and in front of a partially reflective structure with constant water depth}

In this experiment, having $100 \mathrm{~mm}$ water depth in front of the seawall, resulting in a $30 \%$ reflection coefficient, the bed level changes were measured after each run for two different sizes of sediments. Wave conditions corresponding to the $\mathrm{K}$ and $\mathrm{P}$ spectra were used to generate the random waves (Table 1).

The results of the open beach experiments show the coarse sediment beach building a berm, while the finer sand beach erodes to form a long shore bar; whereas the results of the beach experiments in front of the partially reflective seawall show the tendency of the beach to form a berm profile in front of the structure (Fig. 2).

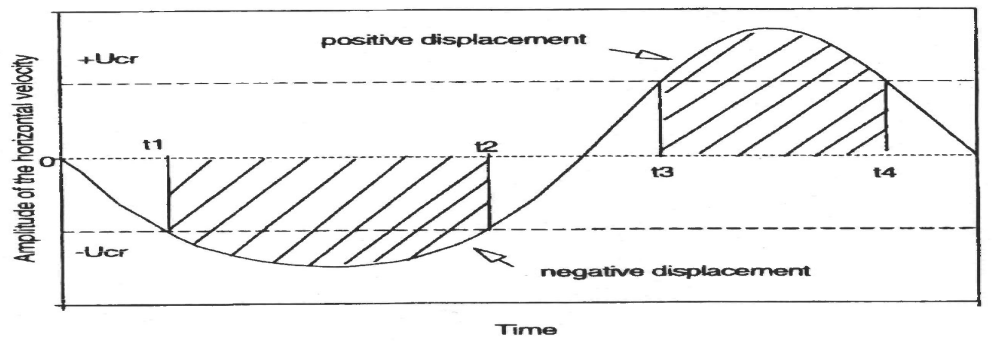

Figure 1: Calculation of sediment displacements based on the time series of horizontal velocity.

Table 1: Descriptions of the spectra used in the experiment. Hs= Significant wave height, $\mathrm{Tz}=$ Zero-crossing period, $\mathrm{L}_{0}=$ Deep water wave length, $\mathrm{S}_{0}=$ Deep water wave steepness.

\begin{tabular}{|c|c|c|c|c|}
\hline Spectrum & $\mathrm{Hs}(\mathrm{m})$ & $\mathrm{Tz}(\mathrm{s})$ & $\mathrm{L}_{0}(\mathrm{~m})$ & $\mathrm{S}_{0}$ \\
\hline $\mathrm{K}$ & 0.080 & 1.0 & 1.56 & 0.051 \\
\hline $\mathrm{P}$ & 0.100 & 1.5 & 3.51 & 0.028 \\
\hline
\end{tabular}

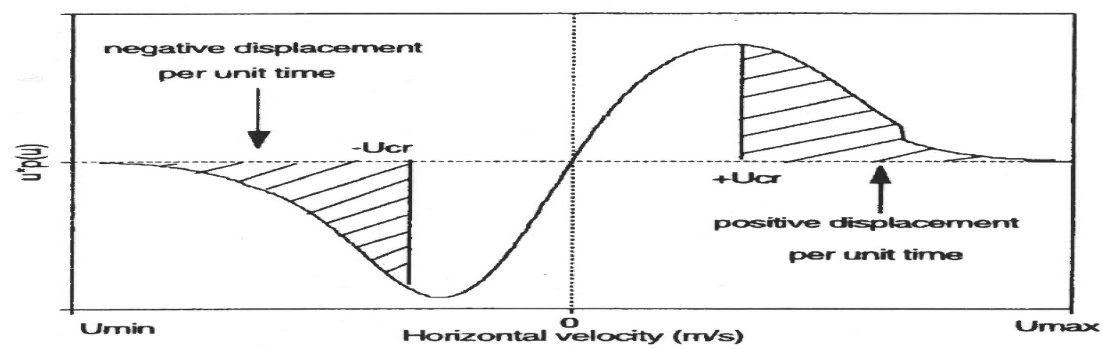

Figure 2: Calculation of sediment displacements based on the probability density function of horizontal velocity. 
This figure also contrasts the changes in bed elevations from the original profiles for both fine and coarse sediments on an open beach with those measured in front of the seawall. Clearly, the experimental results show a different behavior of the beach with respect to the sediment sizes.

\subsection{Measurement of beach development in front of a partially reflective structure with variable water depths}

In the next stage of the experimental programmer, the changes in the beach profile were measured in front of a partially reflective seawall with different water depths to assess the influence of the location of the wall on beach profile evolution. For this purpose, four water depths in front of the seawall were selected $(0,50,100$ and $150 \mathrm{~mm})$ and the changes in bed elevation were measured for three different wave conditions (S1, S2 and S3) summarized in Table 2.

Figure 3 shows the effect of seawall position on sediment transport and beach profile evolution for different wave conditions used in the experiments. Interesting, for both fine and coarse sediments the results show less sediment transport as the water depth in front of the seawall increases. This is in agreement with the results of velocity measurements with variable water levels in front of the structure indicating less offshore-directed flow, undertow, in such cases (Holmes et al [4]).

This figure also indicates that when the water depth in front of the seawall is low, the beach behavior is much the same as a natural beach in which a long shore bar is formed. In summary, the extensive series of tests on the performance

Table 2: Descriptions of the spectra used in the seawall experiment.

\begin{tabular}{|c|c|c|c|c|}
\hline Spectrum & $\mathrm{Hs}(\mathrm{m})$ & $\mathrm{Tz}(\mathrm{s})$ & $\mathrm{L}_{0}(\mathrm{~m})$ & $\mathrm{S}_{0}$ \\
\hline $\mathrm{S} 1$ & 0.074 & 1.24 & 2.40 & 0.031 \\
\hline $\mathrm{S} 2$ & 0.088 & 1.46 & 3.33 & 0.027 \\
\hline $\mathrm{S} 3$ & 0.095 & 1.67 & 4.35 & 0.022 \\
\hline
\end{tabular}

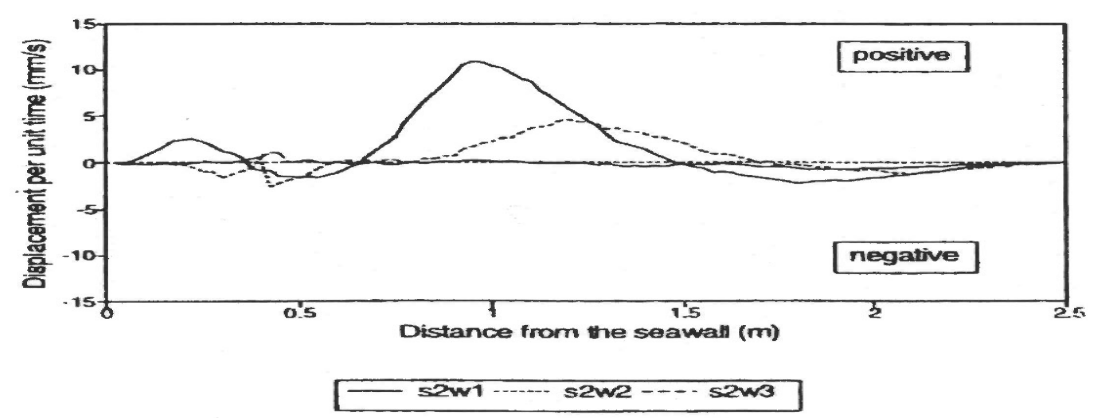

Figure 3: Calculated net sediment displacements across the profile for different wave conditions used in the experiments. w1, w2 and w3 represent $0.05,0.1$ and $0.15 \mathrm{~m}$, water depths at the wall, respectively. 
of a partially seawall on beach evolution clearly show the influence of the structure on the sediment transport and bed level change in the surf zone. While the final shape of the profile was found to be sensitive to the sediment diameter for an open beach, the behavior of the beach in the presence of a partially reflective structure shows much less dependency on the sediment size. On the other hand, the results obtained from the profile evolution measurements in front of a partially reflective structure indicate that the extent to which a seawall affects the processes on the fronting beaches largely depends on the location of the seawall relative to the active shore face. A seawall located well landward of the active shore face will behave in much the same way as a natural beach; whereas seawall located on the active shore face will modify the near-shore beach profile because of the effects of reflected waves.

\section{Model development}

\subsection{Structure of the model (sediment transport)}

A new approach based on the probability density functions of the measured nearbed horizontal velocities is used to develop a semi-empirical model for the calculation of sediment transport and beach profile evolution in the vicinity of a partially reflective structure. The main input to the model is a driving function in the form of a probability density function (PDF) of the near-bed horizontal velocity. A simple sheet flow model is derived to calculate the net sediment transport across the profile in front of a partially reflective structure based on integrating the probability density functions of the near-bed velocities. One of the most important features of the model is the incorporation of a threshold condition for the incipient grain motion in the calculation of sediment displacements. Hallermeier [5] extensively reviewed the different criteria for the initiation of sediment motion. The discrepancies among the criteria were attributed to varying experimental techniques and to the extrapolation of results beyond the range of verification. In oscillatory flow, however, there is no generally accepted relationship for initiation of motion on a plane bed. The most popular equations that are used in the present model and read as:

$$
\begin{array}{cc}
\frac{u_{c r}^{2}}{(s-1) g d_{50}}=0.21\left(\frac{2 A_{c r}}{d_{50}}\right)^{0.5} & : d_{50}<0.0005 \mathrm{~m} \\
\frac{u_{c r}^{2}}{(s-1) g d_{50}}=1.45\left(\frac{2 A_{c r}}{d_{50}}\right)^{0.25} & : d_{50} \geq 0.0005 \mathrm{~m} \\
A_{c r}=\frac{H}{2 \sinh k h} &
\end{array}
$$

in which $U_{c r}=$ critical peak value of orbital velocity near the bed; $A_{c r}=$ critical peak value of orbital excursion near the bed; $\mathrm{D}_{50}=$ the median grain diameter of bed material; $\mathrm{S}=$ specific gravity $\left(\rho_{s} / \rho\right.$ where $\rho_{s}$ and $\rho$ are sediment and fluid density respectively); $\mathrm{h}=$ water depth; $\mathrm{H}=$ wave height; $\mathrm{k}=$ Wave number ( $2 \pi / L$, where $\boldsymbol{L}$ is the wave length); $\mathrm{g}=$ acceleration of gravity

In the present model, a combination of the above and the past equations, which is based on the critical bed shear stress, has been used to estimate the 
critical velocity near the bed - which depends on the sediment properties. As described by Asano [6] and Hoque et al [7], the sheet flow occurs under high shear stress where ripples are washed out. Since sheet flow is a grain-fluid mixture flow of high concentration, the mechanism is more complex than of the other modes of sediment transport. In the sheet flow region where several layers of grains are mobilized, grain to grain collision performs a main role in the momentum exchange. In the high concentration region close to the immovable layer, the sediment transport velocity approaches zero due to the intergranular stress. Following Madsen and Grant [8], Sleath [9] and Dibajnia and Watanabe [10], it is assumed that the sediment in the top layer of the bed moves at the fluid velocity when the threshold velocity for initiation of sediment motion (ucr) is exceeded. Once the grain layer is in motion, it will continue to move in the same direction even when $\mathrm{u}<\mathrm{ucr}$ and it is likely that the mobile grains will decelerate and stop around the point of reversal of the fluid velocity (Fig. 4).

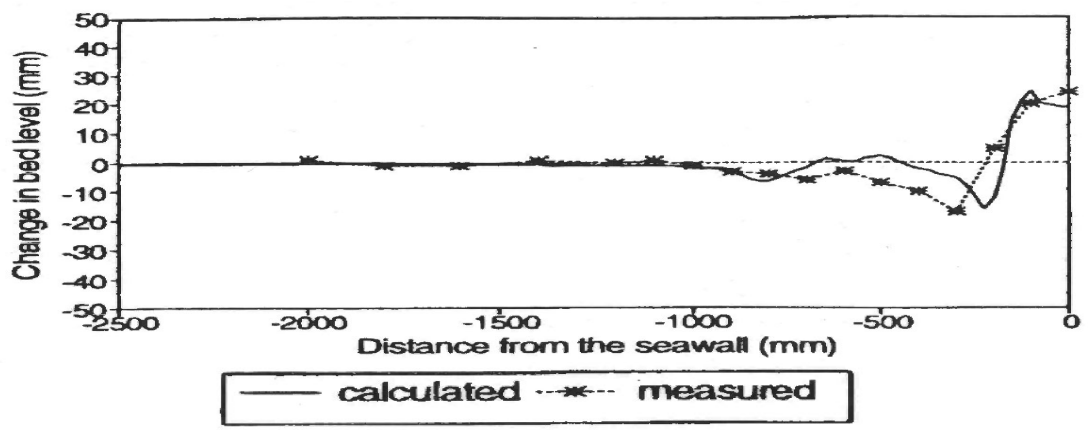

Figure 4: $\quad$ Comparison between predicted and measured bed level changes in front of the partially reflective seawall using a particular Wave condition for coarse sediment.

As a first approximation, this deceleration effect can be ignored and therefore the sediment is assumed to stop moving when $\mathrm{u}<\mathrm{ucr}$. It has to be noted that at this stage the above assumption is used because of its simplicity in the calculation of the net sediment movements. However, more evidence is required to verify this assumption. Referring to Fig. 4, the negative and positive displacements of the sediment can be derived as:

$$
\text { neg . dis. }=\int_{t_{1}}^{t_{2}} u d t \quad \text { pos . dis }=\int_{t 3}^{t} 4 d t
$$

where $\mathrm{U}\left(\mathrm{t}_{1}\right)=\mathrm{U}\left(\mathrm{t}_{2}\right)=-\mathrm{U}_{\mathrm{cr}} ; \mathrm{U}(\mathrm{t} 3)=\mathrm{U}(\mathrm{t} 4)=+\mathrm{U}_{\mathrm{cr}}$

Using this method and adding the successive displacements for the positive and negative components of velocity time series separately, it is possible to calculate the net sediment displacement for a given location during a period of time $(\mathrm{T})$ as:

$$
\text { net .dis . }=\sum_{t=0}^{T}\left(\text { pos .dis .) }-\sum_{t=0}^{T}(\text { neg .dis .) }\right.
$$


In practical situations, however, using the time series of velocity in order to calculate the net sediment displacement is not very convenient. Hence, by transferring the problem to the probability domain, an alternative approach based on the PDF of the velocity time series is introduced to avoid the difficulties associated with time series calculations. This approach can be used for prediction of the gross and net sediment transport rate in the surf zone across the beach. As discussed by Neshaei [11], it is possible to estimate the PDF of the near-bed horizontal velocity based on simulating a Gaussian probability density function and modifying it using a transfer function. According to the definition of the probability density function of horizontal velocity, the shaded area under the PDF in both positive and negative sectors, results represent the percentage of the time in which the velocity of sediment motion exceeds the critical value (ucr). It is assumed that the sediments move with the same velocity as the water particles near the bed. Therefore, the duration of the time that is spent by sediment in positive and negative directions can be estimated, respectively, as follows:

$$
t_{\text {pos }}=T \int_{u_{c r}}^{u_{\max }} p(u) d u \quad t_{n e g}=T \int_{u_{\min }}^{-u_{c r}} p(u) d u
$$

where $\mathrm{T}$ is the total period of the time in which the calculation of sediment displacement is made (duration of storm). According to the basic kinematics equation of motion for a particle, the displacement of a particle in motion can be derived simply as the product of its velocity and the duration of the time in which the particle is in motion. Therefore, the positive and negative displacements can be given, respectively, as:

$$
\text { pos .dis . }=\int_{u}^{u} \max _{c r} \text { up }(u) d u \quad n e g \text {.dis } .=\int_{u_{\min }}^{-u_{c r}} \text { up }(u) d u
$$

The product $U_{p}(u)$ is the first moment of the probability density function of the sediment velocity at the top of the mobile layer. Results show a typical shape of the function $U_{p}(u)$. As can be seen in this figure, for a Gaussian PDF with a zero mean, since the positive and negative displacements are equal for a given ucr, the net displacement will be zero and no net sediment transport will occur in the surf zone. In reality, because of the skewness of the PDF due to nonlinearity and breaking effects in shallow water, the net sediment displacements for each particular location in the surf zone can be calculated as:

$$
\text { netdi. }=\text { pos. dis. }- \text { neg } . \text { dis. }=\int_{u_{c r}}^{u_{\max }} u p(u) d u-\int_{u_{\min }}^{-u_{c r}} u p(u) d u
$$

Records show the net displacements calculated over a number of points across the profile for different wave conditions used in the experiments using the above method. A numerical integration method was applied to calculate the area under the $U_{p}(u)$ curve, which corresponds to the sediment displacement per unit time. As can be seen in this figure, in most cases, inside the surf zone the positive displacements (towards the shoreline) are dominant, resulting in onshore transport and building a berm profile in front of the seawall. These results are in agreement with the experimental observations. 


\subsection{Structure of the model (beach profile development)}

The final feature of the propose model is to calculate the bed level changes and consequently beach profile evolution in front of a partially reflective structure. As described earlier, the positive and negative displacements for a sediment particle per unit time can be calculated by integrating the $U_{p}(u)$ curve using equation 7. By discrediting the profile into a number of segments, it is possible to assign a positive and/or a negative displacement to each section. The next step is to displace each section of the profile into a new position determined by its displacement magnitude. Keeping mind that the displacements calculated by equation 7 are per unit time, it is necessary to repeat this process for each section for the tome in which sediments are in motion. This time can conveniently be calculated from the area under the probability density function of the horizontal velocity for each point across the profile using a numerical integration according to equation 6. By multiplying the time of motion by the volume of each section per unit width of the profile section and transferring the result into the proper location (determined by the magnitude of displacement), i.e. adding the accumulated area, it is possible to calculate the rate of change in bed level across the profile and the final shape of the beach in front of the reflective structure after a given period of time. This assures that the net displacement of sediment from its initial position is sufficiently small so that the ambient velocity field does not change significantly over that displacement. The important query here is how deep the sediments can be eroded in the vertical plane, i.e. what is the exact volume per unit width of each section that is displaced across the profile? To implement this concept into the model, an intrusion depth, defined as the depth to which sediment moves-at the ambient velocity - for sheet flow condition is used to evaluate the vertical length scale of eroded sediments across the profile. The vertical scale of the bed load distribution is defined by:

$$
L_{B}=\frac{1}{c_{\max }} \int_{0}^{\infty} C_{B}(z) d z
$$

in which $\mathrm{C}_{\max }=$ sediment concentration at the top of immobile bed; $\mathrm{C}_{\mathrm{B}}(\mathrm{z})=$ bed load sediment concentration

This can be estimated using Bagnold's assumptions by:

$$
L_{B}=k_{B}\left(\theta^{\prime}-\theta_{C}\right) d
$$

where $\mathrm{d}=$ sediment grain diameter; $\theta^{\prime}=$ skin friction or effective shields parameter (defined by equation 11 ); $\theta_{c}=$ critical Shields parameter $(0.03-0.05$ for practical cases); $\mathrm{k}_{\mathrm{B}}=\mathrm{a}$ constant (suggested to be 2.5 by Bagnold)

The non-dimensional Shields parameter corresponding to the effective bed shear stress is defined by:

$$
\theta^{\prime}=\frac{T^{\prime}}{\rho(s-1) g d}
$$

where $\mathrm{T}^{\prime}=$ the effective bed shear stress; $\rho=$ fluid density

Hence, the area of each section can then be calculated by the product of LB and the length of each section. Finally, by moving this volume per unit width to a 
new location determined by the magnitude of net displacement and considering the time that is spent by each section in motion, it is possible to calculate the profile developments across the beach. Examples of profile evolution results predicted by the present model and comparison of them with those found in experimental investigations are available for both coarse and fine sediments. As can be seen, the predictions are accurate for the lower water levels; as the water depth in front of the wall increases the discrepancies between the predictions and measurements become more significant.

\section{Conclusions}

A sheet flow model is developed to calculate sediment displacement across the profile based on integrating the product of the near-bed horizontal velocities and their probability density functions. Having calculated the sediment displacements, the bed level changes have been estimated based on mass balance at a series of location across the profile. The following conclusions are deduced from the experimental investigations and model development in the present study:

1. The extent to which a seawall affects the processes on the fronting beach largely depends on its location relative to the active shore face. A seawall located well landward of the active shore face will not influence coastal processes expect possibly during periods of exceptionally high water; whereas seawalls located on the active shore face will modify the near-shore beach profile.

2. Taking the instantaneous deviation of the velocity data from the mean into account and incorporating a threshold condition results in a better prediction with respect to the sediment diameter. Noting that the energetic approach is not sensitive to the particle size, the dependency of the predicted results on the sediment diameter is one of the most important advantages of the present model. The results obtained from the profile evolution experiments confirm a strong a strong dependency of the final profile on sediment size.

3. Formulating the sediment transport prediction on the basis of integrating the probability density function of the near-bed horizontal velocity as a driving function to move the sediment provides a strong tool in calculating profile evolution in the surf zone. The validity of this approach has been shown by comparison of the predicted results with those obtained from the experiments.

4. In spite of the sensitivity of the predicted results obtained from the present model to some important elements. Using sheet flow conditions to develop the model as a first approximation is quite reasonable and can produce satisfactory results when compared with the experimental results.

5. The main conceptual innovation of the present work is in taking the probability density functions of the velocities into account and integrating to calculate the sediment displacements across the profile. The experimental results were found to be in fair agreement with those predicted by the model, which is promising and encouraging for further developments of the preliminary model. However, more extensive data are required to establish further the degree of validity of the proposed modeling criteria. 
In summary, although it is not as quantitatively accurate as desired, the preliminary model developed in the present work appears very promising in view of its close qualitative agreement with laboratory investigations. The model is logically based and could be extended to a large variety of naturally occurring wave and sediment conditions. The probabilistic nature employed in the present study is one of the most important advantages of the proposed model. Noting that existing beach morphology models are essentially deterministic, in that they describe the deterministic morphological processes in response to deterministic inputs, the stochastic approach adapted in the present work is an essential contribution to existing knowledge and to the state of the art in modeling coastal morphology.

\section{References}

[1] Rakha, K.A. and Kamphuis, J.W. (1997). A Morphology Model for an Eroding Beach Baked by a Seawall, Coastal Eng., Vol.30, pp.53-75.

[2] Kamphuis, J.W., Rakha, K.A. and Jui, J. (1992). Hydraulic Model Experiments on Seawalls. Proc. of 23rd Int. Conf. on Coastal Eng., ASCE, Vol.2, pp.1272-1284.

[3] Holmes, P. and Neshaei, M.A.L. (1996). The Effect of Seawall on Coastal Morphology. Proc. of the Ecohydraulics 2000. Vol. A, pp. 525-530.

[4] Holmes, P., Baldock, T.E., Chan, R.T.C. and Neshaei, M.A.L. (1996). Beach Evolution under Random Waves. Proc. Of 25th Int. Conf. On Coastal Eng., ASCE, pp. 3006-3019.

[5] Hallermeier, R.J. (1980). Sand Motion Initiation by Water Waves: Two Asymptotes. J. of Waterway, Port, Coastal and Ocean Engineering. ASCE, Vol. 106, No. WW3, pp. 229-318.

[6] Asano, T. (1995). Sediment Transport under Sheet-Flow Conditions. J. of Waterway, Port, Coastal and Ocean Engineering. ASCE. Vol.121, No.5.pp. 239-246.

[7] Hoque, M.A. \& Asano, T. \& Neshaei, M.A.L (2001). Effect of Reflective Structures on Undertow Distribution.' proceeding of the Fourth International Symposium Waves 2001-California-USA, Vol. 2, pp 10421051 .

[8] Madsen, O.S. and Grant, W.D. (1976). Quantification Description of Sediment Transport by Waves. Proc. of 15th Int. Conf. On Coastal Eng., ASCE, pp. 1093-1112.

[9] Sleath J.F.A. (1993). Bed Load Transport in Oscillatory Flow. IAHR, pp. 93-106.

[10] Dibajnia, M. and Watanabe, A. (1992). Sheet Flow under Nonlinear Waves and Currents. Proc. Of 23 rd Int. Conf. On Coastal Eng., ASCE, pp. 2015-2028.

[11] Neshaei, M.A.L. (1997). A Semi-Empirical Model for Beach Profile Evolution in front of a Partially Reflective Structure. Proc. of the XXVII IAHR Congress, ASCE, pp. 31-36. 